\title{
Retrospective Chart Review Comparing CKD COVID-19 Positive Patient Outcomes to non-CKD Patient Outcomes
}

Nicole Eckert ${ }^{1}$, Safiya Sankari², Katie Allen ${ }^{3}$, Siu Lui Hui ${ }^{1,3}$, Eneida Mendonca ${ }^{1,3}$

${ }^{1}$ Indiana University School of Medicine; ${ }^{2}$ Eman School, ${ }^{3}$ Regenstrief Institute

\section{Background/Objective:}

Since January 2020, there have been over 3 million individuals infected with the coronavirus in the United States, quickly spreading across at least 171 countries. The severity and morbidity of patients with COVID-19 are significantly increased when comorbidities, such as Chronic Kidney Disease (CKD), are present. Because the main target of SARS-CoV-2 is ACE2, patients with CKD may be a more vulnerable population. The goal of this study was to determine if COVID-19 positive patients with CKD had increased mortality, inpatient admission, and ED visitation rates compared to those without CKD.

\section{Methods:}

This retrospective chart review includes patients from over 100 separate healthcare entities who were diagnosed with COVID-19 between January 1, 2020 and July 13, 2020 and are over the age of 18. The subjects were first separated into those diagnosed with CKD and those without, basic descriptive calculations were computed, and a Chi Square test was used to analyze outcomes.

\section{Results:}

The CKD COVID-19 positive population was compromised of $47.5 \%$ men and $52.5 \%$ women while the non-CKD control group was made up of $45.4 \%$ men, $54.1 \%$ women, and $0.5 \%$ other. The median Charlson index for the CKD and non-CKD population was 4 and 1, respectively. The interest and control groups were further divided into subpopulations by age and race and analyzed accordingly. Chi square tests demonstrated that there is a statistically significant difference $(p<0.05)$ in all clinical outcomes tested of CKD patients diagnosed with COVID-19 compared to non-CKD patients. The CKD population had increased mortality, inpatient admission, and ED visitation rates when compared.

\section{Discussion:}

This study demonstrates that comorbidities, more specifically CKD, may be associated with a higher severity of COVID-19 than those without. Future studies are needed to explore the relationship more extensively, analyze other outcomes, and manage confounding variables. 